\title{
Efeito da dieta hipoenergética sobre a composição corporal e nível sérico lipídico de mulheres adultas com sobrepeso
}

\author{
Effect of a low-energy diet on the body \\ composition and serum lipid levels \\ of overweight adult women
}

\author{
Valéria Sales do VALLE' ${ }^{1}$ \\ Cíntia BIEHL' \\ Danielli Braga de MELLO2 \\ Marcos de Sá Rego FORTES 3 \\ Estélio Henrique Martin DANTAS ${ }^{4}$
}

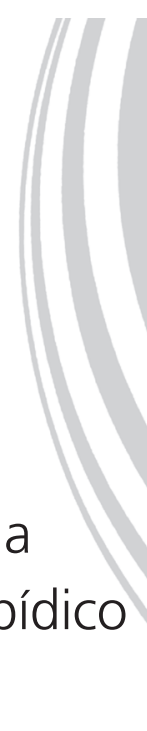

ORIGINAL | ORIGINAL

RE S U M O

\section{Objetivo}

Verificar o efeito de 12 semanas de dieta hipocalórica sobre a composição corporal e o nível sérico lipídico de mulheres adultas com sobrepeso.

\section{Métodos}

A amostra foi composta por vinte mulheres (23,80, desvio-padrão de 2,73 anos) da academia Westfit-Bangu, divididas randomicamente em dois grupos de dez: grupo controle e grupo dieta hipoenergética. Foi realizada uma avaliação da composição corporal (massa corporal, percentual de gordura, índice de massa corporal e massa magra) e do nível sérico lipídico (colesterol total, triglicerídeos, lipoproteína de baixa densidade, lipoproteína de alta densidade e lipoproteínas de muito baixa densidade). Utilizou-se a estatística descritiva (média e desvio-padrão) e o teste $t$ de Student na análise inter e intragrupos. O nível de significância foi de $p<0,05$.

\section{Resultados}

O grupo dieta hipoenergética apresentou reduções significativas $(p<0,05)$ nas variáveis antropométricas (massa corporal, percentual de gordura, índice de massa corporal e massa magra) e nas variáveis lipídicas (triglicerídios,

\footnotetext{
${ }^{1}$ Universidade Castelo Branco, Laboratório de Biociências da Motricidade Humana. Av. Salvador Allende, 6.700, Recreio dos Bandeirantes, 22780-160, Rio de Janeiro, RJ, Brasil, Correspondência para/Correspondence to: V.S.VALLE. E-mail: <valeriasvalle@yahoo.com.br>.

2 Escola de Educação Física do Exército. Urca, RJ, Brasil.

3 Instituto de Capacitação Física do Exército. Urca, RJ, Brasil

${ }^{4}$ Universidade Federal do Estado do Rio de Janeiro, Laboratório de Biociências da Maternidade Humana. Rio de Janeiro, RJ, Brasil.
} 
colesterol total, lipoproteína de baixa densidade e lipoproteínas de muito baixa densidade). Quanto à variável: lipoproteína de alta densidade, foi observada uma redução, porém não significativa.

\section{Conclusão}

A partir dos resultados apresentados pode-se concluir que a dieta hipoenergética foi uma excelente opção no tratamento da obesidade e no controle do nível sérico dos lipídeos, contribuindo desta forma, para a redução dos riscos cardiovasculares na população estudada.

Termos de indexação: Dieta hipoenergética. Lipídeos plasmáticos. Obesidade.

\section{A B S T R A C T}

\section{Objective}

This study verified the effects of a 12-week low-calorie diet on the body composition and serum lipid levels of overweight adult women.

\section{Methods}

The sample consisted of 20 females (23.80 years with a standard deviation of 2.73 years) of the Westfit-Bangu gym. They were randomly divided into two groups of ten: the Control Group and the Diet Group. Body composition (body mass, \%fat, body mass index and lean body mass) and serum lipid levels (total cholesterol, triglycerides and high-, low- and very low-density lipoproteins) were assessed. Descriptive (central tendency and dispersion) and inferential statistics (Student's t-test) were used for inter and intragroup analysis. The significance level was set at $p<0.05$.

\section{Results}

The Diet Group presented a significant decrease $(p<0.05)$ in anthropometric variables (body mass, \% of fat, $B M I$ and lean mass) and serum lipids (triglycerides, total cholesterol and low- and very low-density lipoproteins). The level of high-density lipoprotein also decreased but not significantly.

\section{Conclusion}

In conclusion, the low-calorie diet was an excellent option for the treatment of obesity and to control serum lipid levels, decreasing the cardiovascular risk of the studied population.

Indexing terms: Low-energy. Lipids. Obesity.

\section{N T R O D U Ç Ã O}

Há uma crescente preocupação mundial com o aumento da prevalência de sobrepeso e obesidade (índice de massa corporal $\geq 25 \mathrm{~kg} / \mathrm{m}^{2} \mathrm{e}$ $\geq 30 \mathrm{~kg} / \mathrm{m}^{2}$, respectivamente) ${ }^{1}$. Considerada atualmente como epidemia, a obesidade vem crescendo de forma alarmante tanto nos países desenvolvidos como naqueles em desenvolvimento, acarretando enormes prejuízos à sociedade 2 .

É uma doença crônica caracterizada pelo acúmulo excessivo de gordura corporal, podendo por si só contribuir para o desenvolvimento de comorbidades como diabetes, hipertensão, doenças coronarianas, dislipidemias e outras que contribuem de forma importante para reduzir a qualidade e a expectativa de vida ${ }^{3}$.
É reconhecido que os fatores genéticos têm um papel importante sobre a obesidade, porém é necessário analisar o comportamento e o ambiente em que a família se encontra, pois se estes não forem controlados, podem tornar o indivíduo mais suscetível a essa doença ${ }^{4}$. Acredita-se que os fatores ambientais e comportamentais - sedentarismo e excesso de calorias consumidas - sejam os mais relevantes na incidência da obesidade do que os fatores genéticos ${ }^{5,6}$.

Associadas ao excesso de peso, as dislipidemias aumentam o risco para doenças cardiovasculares ateroscleróticas, que representam importante causa de morbidade e mortalidade no Brasil7. Intervenções para manutenção de peso saudável e valores normais dos lipídeos fazem parte de medidas de prevenção primária das 
doenças cardiovasculares também em indivíduos sem evidências de cardiopatia8.

Das variáveis ambientais envolvidas na determinação do perfil lipídico e da obesidade, uma intervenção dietética é a estratégia mais efetiva tanto para a redução do percentual de gordura quanto para modificações favoráveis do

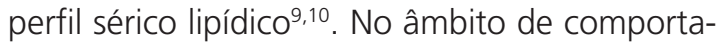
mentos para uma vida saudável, está fortemente relacionada com a promoção da saúde ${ }^{11}$.

Os hábitos alimentares influenciam de forma marcante o balanço energético positivo. A transição nutricional que vem acontecendo nos últimos anos apresenta características próprias em cada país; no entanto, elementos comuns convergem para a chamada dieta ocidental, que consiste em uma alimentação rica em gorduras, sobretudo as de origem animal, açúcar e alimentos refinados além de reduzida ingestão de carboidratos complexos e fibras, que favorecem o estoque energético $^{12}$.

Um comportamento nutricional adequado é imprescindível tanto para uma boa saúde como para um bom desempenho físico. As quantidades de macronutrientes disponíveis na dieta devem satisfazer as necessidades fisiológicas normais dos indivíduos sadios ou não, da forma mais equilibrada possível, prevenindo o surgimento de doenças $^{13}$.

Há cerca de quarenta anos começou a ser dada maior ênfase ao papel da dieta na saúde e enfermidades crônico-degenerativas, como as doenças cardiovasculares, dislipidemias, diabetes Mellitus, diferentes tipos de câncer e obesidade ${ }^{14}$.

Com isso a presente pesquisa teve como objetivo verificar o efeito de 12 semanas de dieta hipoenergética sobre a composição corporal e o nível sérico lipídico de mulheres adultas com sobrepeso.

\section{MATERIAIS E MÉTODOS}

A presente investigação caracteriza-se como uma pesquisa com delineamento quase experimental.
A amostra foi composta por vinte sujeitos do sexo feminino (Média $(M)=23,80$ Desvio-Padrão (DP)=2,73 anos), aparentemente saudáveis, de acordo com American College of Sports Medicine $(\mathrm{ACSM})^{15}$, voluntárias e iniciantes em um programa de ciclismo indoor. Foram divididas randomicamente em dois grupos: Grupo dieta hipoenergética (GD) e Grupo Controle (GC). Cada grupo foi constituído por dez indivíduos.

O presente trabalho atendeu às normas para a realização de pesquisa em seres humanos, resolução 196/96 do Conselho Nacional de Saúde de 10 de outubro de $1996^{16}$

Todas as voluntárias foram informadas oralmente e por escrito sobre os procedimentos do presente estudo, e concordaram em assinar um Formulário de Consentimento. Este trabalho foi avaliado e aprovado pelo Comitê de Ética em Pesquisa da Universidade Castelo Branco - UCB/ Rio de Janeiro, Brasil; protocolo nº 0019/2008.

A coleta de dados foi dividida em quatro etapas:

$1{ }^{\circ}$ Etapa: Avaliação da Composição Corporal: Foi mensurada por meio de métodos antropométricos conforme o protocolo citado por Lohman et al. ${ }^{17}$. Foram medidas as seguintes variáveis: Massa Corporal (MC), Estatura, Índice de Massa Corporal (IMC), Percentual de Gordura (\%G) e Massa Magra (MM).

As medidas de massa corporal $(\mathrm{kg})$ e estatura $(m)$ foram realizadas em uma balança (Filizola ${ }^{\circledR}$, Brasil) com precisão de $100 \mathrm{~g}$ e escala de 0 a $150 \mathrm{~kg}$, a qual possuía um estadiômetro acoplado.

O índice de massa corporal (IMC), obtido pela divisão dessas duas medidas $\left(\mathrm{kg} / \mathrm{m}^{2}\right)$, foi utilizado para a classificação da obesidade segundo os limites de corte recomendados pela OMS'.

O percentual de gordura foi verificado segundo o protocolo de sete dobras de Jackson e Pollock \& Wilmore ${ }^{18}$ através de um plicômetro $\left(\right.$ Lange $\left.^{\circledR}\right)$. 
$2^{\circ}$ Etapa: Avaliação diagnóstica: Foi realizada por um laboratório de análises clínicas e mensurada através da coleta sanguínea as seguintes variáveis: Triglicerídeos (TG), lipoproteína de baixa densidade $(L D L)$, lipoproteína de muito baixa densidade (VLDL), lipoproteína de alta densidade (HDL) e Colesterol Total (CT). Para as dosagens da HDL, CT e TG, foi utilizado o método enzimático colorimétrico. O VLDL e LDL foram calculados pela equação de FRIEDEWALD sendo todos os valores expressos em $\mathrm{mg} / \mathrm{dl}$.

As duas etapas da coleta ocorreram na mesma semana.

\section{$3^{\circ}$ Etapa: Intervenção}

Dieta Hipocalórica: O grupo GD foi orientado por uma nutricionista através de um recordatório alimentar. Durante o período de estudo, os indivíduos participaram de reuniões quinzenais. Em todos os atendimentos, além da avaliação nutricional, foram verificadas quais das metas estabelecidas foram efetivamente alcançadas. A seguir, as participantes tinham a oportunidade de expressar as dificuldades sentidas para seguir as orientações da(s) consulta(s) anterior(es), sendo, então, orientadas em relação às possíveis formas para contornar tais dificuldades.

O consumo energético foi de aproximadamente 1 200kcal/dia, divididas em aproximadamente oito refeições (com intervalo de no mínimo duas horas e no máximo quatro horas). Os indivíduos foram orientados a não substituir as refeições principais por lanches, respeitar sempre os horários e quantidades e ingerir no mínimo dois litros de água por dia. O período compreendido para essa restrição foi de 12 semanas.

Cada indivíduo recebeu além do plano alimentar com uma lista de substituição para eventual troca (sempre respeitando as quantidades), observações e informações sobre a rotina alimentar.

A ingestão de gorduras saturadas foi limitada, por constituírem o principal fator determinante da elevação das concentrações plasmáticas de LDL. Em seguida, os lipídeos que mais contri- buem para esse aumento que são os ácidos graxos trans isômeros, e em menor grau, o colesterol dietético ${ }^{19}$.

$4^{\circ}$ Etapa: Reavaliação

Ao final de 12 semanas de intervenção o grupo experimental realizou os mesmos procedimentos e protocolos descritos na $1^{\circ}$ e $2^{\circ}$ etapas.

Para análise dos resultados utilizou-se a estatística descritiva ${ }^{20}$ por meio de medidas de localização (média) e de dispersão (desvio-padrão). A homogeneidade das amostras foi verificada através da Curtose, e a normalidade através do teste de Kolmogorov-Smirnov.

Foram utilizadas técnicas de estatística inferencial para comparação entre as médias através do teste $t$ de Student na análise inter e intragrupos. O nível de significância adotado foi de $95 \%(p \leq 0,05)$.

\section{RES U L T A D O S}

Na Tabela 1 estão expostos os resultados descritivos das variáveis antropométricas dos grupos controle (GC) e dieta hipoenergética (GD).

Nesta verifica-se que todos os grupos apresentaram uma distribuição normal e homogênea para todas as variáveis analisadas além de um grau de sobrepeso (IMC $\left.\geq 25,0 \mathrm{~kg} / \mathrm{m}^{2}\right)$. A curtose foi realizada para assegurar a homogeneidade da amostra.

Na Tabela 2 estão expostos os resultados descritivos das variáveis do nível sérico lipídico dos grupos controle (GC) e dieta hipoenergética (GD).

A partir dos dados acima podemos observar que ambos os grupos apresentaram uma distribuição normal e homogênea para todas as variáveis antropométricas e do nível sérico lipídico.

Na Tabela 3 estão expostos os resultados das variáveis antropométricas dos grupos GC e GD antes e após 12 semanas de intervenção.

Após 12 semanas, o grupo GD apresentou reduções significativas na massa corporal $(p=0,00)$, percentual de gordura $(p=0,00), \operatorname{IMC}(p=0,00)$ e 
massa magra $(p=0,00)$. Ao contrário do grupo GD, o grupo GC apresentou um aumento significativo nas médias das variáveis $M C(p=0,00)$ e $\% G$ $(p=0,00)$. No entanto, não foram observadas alterações no IMC e MM.

De acordo com os critérios de obesidade e sobrepeso os grupos GC e GD continuaram classificados como sobrepeso. Ao compararmos os dois grupos entre si não foram observadas diferenças significativas $(p>0,05)$.

Na Tabela 4 estão expostos os resultados das médias das variáveis do nível sérico lipídico dos grupos GC e GD antes e depois de 12 semanas de intervenção.

O grupo GD apresentou alterações positivas nas médias das variáveis. Houve uma diminuição no nível plasmático do TG $(-6,5 \mathrm{mg} / \mathrm{dL})$, CT (-10,6mg/dL), LDL (-6,7mg/dL), VLDL (-3,1 mg/dL). A média da variável $H D L$ sofreu redução $(-1,2 \mathrm{mg} / \mathrm{dL})$, porém não foi estatisticamente significativo.

Segundo os valores de referência dos lipídeos plasmáticos recomendados pela IV diretriz brasileira sobre dislipidemias e diretriz de preven-

Tabela 1. Resultados descritivos das variáveis antropométricas dos grupos GC e GD.

\begin{tabular}{|c|c|c|c|c|c|c|}
\hline Idade & & Estatura $(m)$ & $\mathrm{MC}(\mathrm{kg})$ & $\%$ G & IMC $\left(k g / m^{2}\right)$ & $\mathrm{MM}(\mathrm{kg})$ \\
\hline KS & 1,00 & 0,71 & 0,96 & 0,94 & 0,97 & 0,48 \\
\hline GD & $M=23,50 \mathrm{DP}=1,78$ & $M=1,61 \quad D P=0,03$ & $M=71,43 \quad D P=4,22$ & $M=33,09 D P=3,65$ & $M=27,60 \quad D P=1,54$ & $M=47,68 \mathrm{DP}=1,70$ \\
\hline KS & 0,77 & 0,96 & 0,90 & 0,99 & 0,10 & 0,84 \\
\hline
\end{tabular}

GC: grupo controle; GD: grupo dieta; MC: massa corporal; \%G: percentual de gordura; IMC: índice de massa corporal; MM:massa magra; KS: Kolmogorov-Smirnov. Curt: curtose; M: média; DP: desvio-padrão.

Tabela 2. Resultados descritivos do nível sérico lipídico dos grupos GC e GD.

\begin{tabular}{lccccc}
\hline & $T G$ & $T$ & $L D L$ & $H D L$ & VLDL \\
\hline$G C$ & $M=98,10 D P=6,52$ & $M=173,30 D P=10,89$ & $M=114,80 D P=10,72$ & $M=41,20 D P=2,57$ & $M=17,30 D P=3,50$ \\
KS & 0,98 & 0,62 & 0,32 & 0,94 & 0,64 \\
Curt & 1,35 & 0,61 & 0,03 & 0,82 & 2,11 \\
GD & $M=102,60 \mathrm{DP}=6,72$ & $M=172,90 \mathrm{DP}=10,91$ & $\mathrm{M}=111,70 \mathrm{DP}=11,04$ & $\mathrm{M}=42,20 \mathrm{DP}=2,10$ & $\mathrm{M}=19,00 \mathrm{DP}=2,26$ \\
KS & 0,93 & 0,80 & 0,89 & 0,99 & 0,33 \\
Curt & $-1,32$ & 1,58 & 1,63 & $-0,25$ & 0,76 \\
\hline
\end{tabular}

TG: triglicerídeos; CT: colesterol; LDL: lipoproteína de baixa densidade; HDL: lipoproteína de alta densidade; VLDL: lipoproteína de muito baixa densidade; KS: Kolmogorov-Smirnov; Curt: curtose; M: média; DP: desvio-padrão.

Tabela 3. Comparação entre as médias antropométricas dos grupos GC e GD.

\begin{tabular}{|c|c|c|c|c|c|c|c|c|}
\hline \multirow{3}{*}{ Variáveis } & \multicolumn{4}{|c|}{ Grupo controle (GC) } & \multicolumn{4}{|c|}{ Grupo experimental (GD) } \\
\hline & \multicolumn{2}{|c|}{ Pré } & \multicolumn{2}{|c|}{ Pós } & \multicolumn{2}{|c|}{ Pré } & \multicolumn{2}{|c|}{ Pós } \\
\hline & $\mathrm{M}$ & $\mathrm{DP}$ & M & $\mathrm{DP}$ & M & $\mathrm{DP}$ & M & DP \\
\hline$\%$ G & 31,66 & 3,15 & 32,09 & 3,08 & 33,09 & 3,65 & 30,33 & $3,51^{*}$ \\
\hline $\mathrm{IMC}\left(\mathrm{kg} / \mathrm{m}^{2}\right)$ & 27,52 & 1,68 & 27,77 & 1,56 & 27,60 & 1,54 & 25,26 & $1,59^{*}$ \\
\hline
\end{tabular}

M: média; DP: desvio-padrão; MC: massa corporal; \%G: percentual de gordura; IMC: índice de massa corporal; MM: massa magra. ${ }^{*} p<0,05$. 
Tabela 4. Comparação entre as médias do nível sérico lipídico dos grupos GC e GD.

\begin{tabular}{|c|c|c|c|c|c|c|c|c|}
\hline \multirow{3}{*}{ Variáveis } & \multicolumn{4}{|c|}{ Grupo controle (GC) } & \multicolumn{4}{|c|}{ Grupo experimental (GD) } \\
\hline & \multicolumn{2}{|c|}{ Pré } & \multicolumn{2}{|c|}{ Pós } & \multicolumn{2}{|c|}{ Pré } & \multicolumn{2}{|c|}{ Pós } \\
\hline & M & $\mathrm{DP}$ & $M$ & $\mathrm{DP}$ & $\mathrm{M}$ & DP & $M$ & $\mathrm{DP}$ \\
\hline TG & 98,10 & 6,52 & 98,90 & 5,86 & 102,60 & 6,72 & 96,10 & $5,45^{*}$ \\
\hline CT & 173,30 & 10,89 & 175,80 & 11,21 & 172,90 & 10,91 & 162,30 & $10,42^{*}$ \\
\hline LDL & 114,80 & 10,72 & 116,60 & 10,85 & 111,70 & 11,04 & 105,00 & $11,08^{*}$ \\
\hline $\mathrm{HDL}$ & 41,20 & 2,57 & 41,50 & 2,27 & 42,20 & 2,10 & 41,40 & 1,84 \\
\hline VLDL & 17,30 & 3,50 & 17,70 & 2,91 & 19,00 & 2,26 & 15,90 & $1,60^{*}$ \\
\hline
\end{tabular}

M: média; DP: desvio-padrão; TG: triglicerídeos; CT: colesterol; LDL: lipoproteína de baixa densidade; HDL: lipoproteína de alta densidade; VLDL: lipoproteína de muito baixa densidade. ${ }^{*} p<0,05$.

ção da aterosclerose do departamento de aterosclerose da sociedade brasileira de cardiologia ${ }^{21}$, os grupos GC e GD apresentaram-se na categoria ótimo nas seguintes variáveis: TG, CT, HDL e VLDL. Quanto ao LDL, foram classificados como desejável em ambos os grupos.

Na análise intergrupos não foi observada diferença significativa em nenhuma variável $(p>0,05)$.

\section{I S C U S S Ã O}

Após 12 semanas de intervenção dietética, o grupo GD manteve-se na mesma categoria para todas as variáveis lipídicas, porém apresentou modificações positivas e significativas, tais como redução do TG, CT, LDL e VLDL. Segundo Wood et al. ${ }^{22}$ a dieta hipocalórica é considerada imprescindível no tratamento das dislipidemias. Com isso, pode-se dizer que a restrição dietética foi importante para se obter tais alterações.

Estudos demonstram que a dieta exerce um efeito negativo sobre a $H D L$, uma vez que reduzem seus níveis plasmáticos ${ }^{23,24}$. A atual pesquisa corrobora com os estudos acima, pois foi observada uma redução significativa no grupo GD.

Já está bem consolidado na literatura que alterações dietéticas que visem à diminuição dos níveis de LDL constituem a primeira estratégia que deve ser utilizada a nível populacional para a redução do risco cardiovascular ${ }^{25}$.

A ingestão calórica excessiva, com elevado teor de gordura e colesterol, está associada a níveis séricos aumentados de colesterol total (CT) e fração de colesterol da lipoproteína de baixa densidade - LDL ${ }^{26,27}$. Em adultos, concentração aumentada de CT e diminuída da fração de colesterol da lipoproteína de alta densidade (HDL), hipertensão arterial, tabagismo, diabetes e obesidade estão associados a lesões avançadas de aterosclerose e maior risco de manifestações clínicas da doença aterosclerótica ${ }^{28}$.

Fornes et al..$^{29}$ ao estudarem a relação entre a frequência de consumo de alimentos e os níveis séricos de lipoproteínas em população do município de Cotia, SP, observaram que o controle dietético pode reduzir os riscos de doenças cardiovasculares. Neste mesmo estudo, o consumo de carnes processadas, aves, carnes vermelhas, ovos e leite/derivados correlacionaram-se positiva e significantemente com as frações LDL-colesterol, enquanto o consumo de frutas e hortaliças mostrou correlação inversa.

Quanto às variáveis antropométricas, os grupos GC e GD apresentaram valores médios para a variável IMC dentro da faixa que os permite classificá-los com grau de sobrepeso (Media $(M)=27,52$, Desvio-Padrão (DP) $=1,68 \mathrm{~kg} / \mathrm{m}^{2} \mathrm{e}$ $M=27,60, D P=1,54 \mathrm{~kg} / \mathrm{m}^{2}$, respectivamente).

Ao analisarmos os resultados da variável $\% \mathrm{G}$, segundo os padrões de normalidade para percentual de gordura proposto por Pollock \& Wilmore ${ }^{18}$, podemos classificar os grupos GC e GD como ruim ( $M=31,66, D P=3,15 \%$ e $M=33,09$, $\mathrm{DP}=3,65 \%$, respectivamente). 
A prevalência de sobrepeso e obesidade triplicou no Brasil nas últimas décadas ${ }^{30}$. Entre as mulheres da Região Sudeste só apresentou elevação entre aquelas que compõem os $25,0 \%$ mais pobres, passando de $11,6 \%$ em 1989, para $15,0 \%$ em $1997^{31}$. Estimativas mostram que, em 2025, o Brasil será o quinto país a ter problemas com a obesidade em sua população. Nossos resultados já sugerem este crescente aumento de indivíduos com excesso de peso.

Os resultados encontrados no grupo GD após 12 semanas evidenciam a importância de modificações dietéticas no combate à obesidade. Foi observada uma redução significativa nas médias das variáveis MC, IMC e \%G. Segundo Bernardi et al. ${ }^{32}$, a perda da massa corporal alcançada somente pela restrição dietética leva à meIhoria de todo o quadro patológico associado à obesidade.

O grupo GD reduziu significativamente a massa magra corroborando com a afirmativa de que a dieta, isoladamente pode acarretar uma redução na $\mathrm{MM}$ e consequentemente na taxa metabólica de repouso (TMR), visto que, a MM é a variável que mais contribui para o metabolismo energético ${ }^{33}$.

Klein et al. ${ }^{34}$ observaram que indivíduos com sobrepeso ou obesidade, dietas hipoenergéticas que variam entre $800-1500 \mathrm{kcal} / \mathrm{dia}$ reduzem aproximadamente $8 \%$ do peso corporal após um período de seis meses de tratamento dietético. Estes dados corroboram a presente pesquisa, onde os indivíduos que foram submetidos a um consumo energético de $1200 \mathrm{kcal} / \mathrm{dia}$ reduziram em $8,5 \%$ do peso corporal, porém o tempo de intervenção foi menor (três meses). As dietas muito restritas em energia, cerca de $<800 \mathrm{kcal} / \mathrm{dia}$, não são recomendadas para uma perda de peso em longo prazo ${ }^{35}$.

Utter et al..$^{36}$ observaram o efeito da dieta e do exercício em mulheres obesas por 12 semanas. A amostra foi dividida em quatro grupos: Dieta (D), Dieta + Exercício (ED), Exercício (E), Controle (C). Os grupos D e ED reduziram a massa corporal e o percentual de gordura, enquanto os grupos E e C não apresentaram mudanças significativas. Tais achados corroboram com a atual pesquisa e ratificam a importância da dieta no controle da massa corporal.

Um estudo de meta-análise realizado por Miller et al. ${ }^{37}$ determinou a efetividade da dieta, do exercício e da dieta associada ao exercício físico na perda de peso. As diferenças relativas à perda de peso através de dieta, exercício físico e dieta associada ao exercício físico foi de $M=10,7$, $D P=0,5 ; M=2,9, D P=0,4$ e $M=11,0, D P=0,6 k g$, respectivamente.

O excesso de gordura corporal está relacionado a alterações desfavoráveis no perfil lipídico, independentemente da idade e do sexo, valores mais altos de índice de massa corporal (IMC) com níveis plasmáticos mais elevados de TG, CT e colesterol não-HDL, e com níveis mais baixos de colesterol $\mathrm{HDL}^{38}$. Indivíduos obesos, especialmente com predomínio da gordura visceral, apresentam valores maiores de triglicerídeos de jejum ${ }^{39}$ e menores de $\mathrm{HDL}^{40}$.

Por outro lado, está também demonstrado que a redução do peso tem consequências favoráveis no perfil lipoproteico ${ }^{41,42}$ e a magnitude da melhora do perfil lipídico obtida pela dieta varia substancialmente entre os indivíduos ${ }^{43}$.

\section{O N C L U S Ã O}

Ao se analisarem os resultados, o grupo GD apresentou reduções significativas nas variáveis antropométricas: MC, IMC e \% G; e consequentemente nas variáveis do nível sérico lipídico: TG, CT, LDL e VLDL. A partir desses, pode-se concluir que, para a população estudada, esse estudo reforça a recomendação de que a primeira conduta a ser adotada para o controle das variáveis antropométricas bem como dos lipídeos plasmáticos deve ser a intervenção dietética.

\section{COLABORADORES}

V.S. VALLE responsável pela elaboração do trabalho. C. BIEHL contribuiu na coleta de dados e na 
elaboração da discussão. D.B. MELLO responsável pela estatística e contribuiu na elaboração dos resultados e a conclusão e co-orientadora da dissertação. M.S.R. FORTES contribuiu na elaboração da introdução, assim como na correção total do artigo. E.H.M. DANTAS responsável pela elaboração do tema bem como os materiais e métodos e orientador da dissertação.

\section{REFERÊ NCIAS}

1. World Health Organization. Obesity: preventing and managing the global epidemic. Geneva: World Health Organization; 1998.

2. López-Fontana CM, Sánchez-Villegas A, MartínezGonzalez MA, Martinez JA. Daily physical activity and macronutrient distribution of low-calorie diets jointly affect body fat reduction in obese women. Appl Physiol Nutr Metab. 2009; 34(4):1-8. doi: 10.1139/H09-015

3. Baptista MN, Vargas JF, Baptista ASD. Depressão e qualidade de vida em uma amostra brasileira de obesos mórbidos. Aval Psicol. 2007; 7(2):235-47.

4. Devlin MJ, Goldfein JA, Petkova E, Jiang H, Raizamn PS, Wolk S, et al. Cognitive behavioral therapy and fluoxetine as adjuncts to group behavioral therapy for binge eating disorder. Obesity Research. 2007; 13(6):1077-88. doi: 10.1038/oby.2005.126.

5. Romero CEM, Zanesco A. O papel dos hormônios leptina e grelina na gênese da obesidade. Rev Nutr. 2006; 19(1):85-91. doi: 10.1590/\$1415-5273200 6000100009.

6. Christakis NA, Fowler JH, The Spread of Obesity in a Large Social Network over 32 Years. New England J Med. 2007; 357(4):370-9.

7. Krause MP, Hallage T, Micukis CP, Gama MPR, Silava SG. Análise do perfil lipídico de mulheres idosas em Curitiba - Paraná. Arq Bras Cardiol. 2008; 90(5): 327-32. doi: 10.1590/S0066-782X20080005000 04.

8. Hubert HB, Feinleib M, Mcnamara PM, Castelli WP. Obesity as an independent risk factor for cardiovascular disease: a 26-year follow-up of participants in the Framingham Heart Study. Circulation. 1983; 67(5):968-77.

9. Kavouras SA, Panagiotakos DB, Pitsavos C, Chrysohoou C, Anastasiou CA, Lentzas Y, et al. Physical activity, obesity status, and glycemic control: the ATTICA study. Med Sci Sports Exerc. 2007; 39(4):606-11. doi: 10.1249/mss.0b013e 31803084eb.

10. Tsintsifa E, Faxantidis P, Tsiligkiroglou-Fachantidou A, Deligiannis A. Interactions among habitual physical ativity, eating patterns, and diet composition. Angiology. 2006; 57(2):205-9. doi: 10.1177/000331970605700211.

11. Arnaiz MG. La emergencia de las sociedades obesogénicas o de la obesidad como problema social. Rev Nutr. 2009; 22(1):5-18. doi: 10.1590/S 1415-52732009000100001.

12. Oliveira AMA, Cerqueira EMM, Souza JS, Oliveira AC. Sobrepeso e obesidade infantil: influência de fatores biológicos e ambientais em Feira de Santana, BA. Arq Bras Endocrinol Metab. 2003; 47(2):144-50. doi: 10.1590/50004-2730200300 0200006.

13. Paschoal VCP, Amâncio OMS. Nutritional Status of Brazilian Elite Swimmers. Int J Sport Nutr Exerc Metab. 2004; 14(1):81-94.

14. Lima FEL, Menezes TN, Tavares MP, Szarfarc SC, Fisverg RM. Ácidos graxos e doenças cardiovasculares: uma revisão. Rev Nutr. 2000; 13(2):73-80. doi: 10.1590/S1415-52732000000200001.

15. American College of Sports Medicine - ACSM. Diretrizes do ACMS para os testes de esforço e sua prescrição. $6^{a}$ ed. Rio de Janeiro: Guanabara Koogan; 2003.

16. Brasil. Resolução CNS 196/96. Conselho Nacional de Saúde, 1996. Diário Oficial da União. 199616 out; n.201.

17. Lohman TG, Roche AF, Martorell R. Anthropometric standardization reference manual. Champaign: Human Kinetics Books;1988

18. Pollock ML, Wilmore JH. Exercícios na saúde e na doença: avaliação e prescrição para prevenção e reabilitação. $2^{a}$ ed. Filadélfia: MEDSI; 1993.

19. Americam Diabetes Association. Clinical practice recommendations. Diabetes Care. 2004, 27(1): S1-S143.

20. Triola MF. Introdução à estatistica. $7^{a}$ ed. Rio de Janeiro: Livros Técnicos e Científicos Editora; 1999.

21. Sociedade Brasileira de Cardiologia. IV Diretriz brasileira sobre dislipidemias e diretriz de prevenção da aterosclerose do departamento de aterosclerose da sociedade brasileira de cardiologia. Arq Bras Cardiol. 2007; 88(Supl.1):1-19.

22. Wood RJ, Volek JS, Liu Y, Shachter NS, Contois JH, Fernandez ML. Carbohydrate restriction alters lipoprotein metabolism by modifying VLDL, LDL, and HDL subfraction distribution and size in overweight men. J Nutr. 2006; 136(2):384-9.

23. Cambri LT, Décimo JP, Souza M, Oliveira FR, Gevaerd MS. Efeito agudo e crônico do exercício físico no perfil glicêmico e lipídico em diabéticos tipo 2 . Motriz. 2007; 13(4):238-48. doi:10.1016/50026-0 495. 
24. Nieman DC, Brock DW, Butterworth D, Utter AU, Nieman CC. Reducing diet and/or exercise training decreases the lipid and lipoprotein risk factors of moderately obese women. J Am Coll Nutr. 2002; 21(4):344-50.

25. Hooper L, Summerbell CD, Higgins JP, Thompson $R L$, Capps NE, Smith GD, et al. Dietary fat intake and prevention of cardiovascular disease: systematic review. BMJ. 2001; 322 (7289):757-63. doi:10.1136/bmj.322.7289.757.

26. Grundy SM. Dietary therapy of hyperlipidemia. In: Gabello WJ. Slide atlas of lipid disorders. $3^{\text {rd }}$ ed. New York: Gower Medical Publishing; 1990.

27. Coelho VG, Caetano LF, Liberatore Júnior RR, Cordeiro JA, Souza DRS. Perfil lipídico e fatores de risco para doenças cardiovasculares em estudantes de medicina. Arq Bras Cardiol. 2005; 85(1):57-62. doi: 10.1590/S0066-782X2005001400011.

28. McGill Jr HC, McMahan CA, Zieske AW, et al. Origin of atherosclerosis in childhood and adolescence. Am J Clin Nutr. 2000; 72(5):1307-15.

29. Fornes NS, Martins IS, Hernan M, VelasquezMelendez G, Ascherio A. Food frequency consumption and lipoproteins serum levels in the population of an urban area, Brazil. Rev Saúde Pública. 2000; 34(4): 380-7.

30. Wang Y, Monteiro CA, Popkin BM. Trends of obesity and underweight in older children and adolescents in the United States, Brazil, China, and Russia. Am J Clin Nutr. 2002; 75(6):971-7. doi: 10.1 590/S0004-27302003000200001.

31. Monteiro CA, Conde W. A tendência secular da obesidade segundo estratos sociais: Nordeste e Sudeste do Brasil, 1975-1989-1997. Arq Bras Endocrinol Metab. 1999; 43(3):186-94. doi: 10.1 590/S1415-52732006000500001.

32. Bernardi F, Cichelero C, Vitolo MR. Comportamento de restrição alimentar e obesidade. Rev Nutr. 2005; 1(18):85-93. doi: 10.1590/S1415-527320050001 00008.

33. Stiegler $P$, Cunliffe $A$. The role of diet and exercise for the maintenance of fat-free mass and resting metabolic rate during weight loss. Sports Med. 2006; 36(3):239-62. doi:10.2165/00007256200636030-00005.

34. Klein S, Burke LE, Bray GA, Blair S, Allison DB, PiSunyer $X$, et al. Clinical implications of obesity with specific focus on cardiovascular disease. A Statement for professionals from the American
Heart Association council nutrition, physical activity, and metabolism. Circulation. 2004; 110(18):2952-67. doi: 10.1161/01.

35. Steemburgo T, Dall'Alba V, Gross JL, Azevedo MJ. Fatores dietéticos e síndrome metabólica. Arq Bras Endocrinol Metab. 2007; 51(9):1425-33. doi: 10.1 590/S0004-27302007000900004.

36. Utter AC, Nieman DC, Shannonhouse EM, Butterworth DE, Nieman C N. Influence of diet and/ or exercise on body composition and cardiorespiratory fitness in obese women. Int J Sport Nutr. 1998: 8(3):213-22.

37. Miller WC, Koceja DM, Hamilton EJ. A metaanalysis of the past 25 years of weight loss research using diet, exercise or diet plus exercise intervention. Inter J Obes. 1997; 21(10):941-7.

38. Brown CD, Higgins $M$, Donato KA, Rohde FC, Garrison R, Obarzanek E, et al. Body mass index and the prevalence of hypertension and dyslipidemia. Obes Res. 2000; 8(9):605-19. doi: 10.1038/oby. 2000.79.

39. Kissebah AH, Vydelingum N, Murray R, Evans DJ, Hartz AJ, Kalkhoff RK, et al. Relation of body fat distribution to metabolic complications of obesity. J Clin Endocrinol Metab. 1982; 54(2):254-60.

40. Desprès JP, Allard C, Tremblay A, Talbot J, Bouchard C. Evidence for regional component of body fatness in the association with serum lipids in men and women. Metabolism. 1985; 34(10):967-73.

41. Wood PD, Stefanick ML, Dreon DM, Frey-Hewitt B, Garay SC, Williams PT, et al. Changes in plasma lipids and lipoproteins in overweight men during weight loss through dieting as compared to exercise. NEJM. 1988; 319(18):1173-9.

42. Dixon JB, O'Brien PE. Lipid profile in the severely obese: changes with weight loss after lap-band surgery. Obes Res. 2002; 10(9):903-10. doi: 10.1038/oby.2002.

43. Schaefer EJ, Lamon-Fava S, Ausman LM, Ordovas JM, Clevidence BA, Judd JT, et al. Individual variability in lipoprotein cholesterol response to National Cholesterol Education Program Step 2 diets. Am J Clin Nutr. 1997; 65(3):823-30.

Recebido em: 10/1/2009

Versão final reapresentada em: 27/5/2010 Aprovado em: 15/7/2010 
